\title{
Comet 240P/NEAT Is Stirring
}

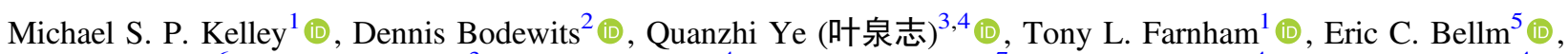 \\ Richard Dekany ${ }^{6}$, Dmitry A. Duev ${ }^{3}$ (D), George Helou ${ }^{4}$ (D), Thomas Kupfer ${ }^{7}$ (D), Russ R. Laher ${ }^{4}$ (D), Frank J. Masci ${ }^{4}$ (D), \\ Thomas A. Prince ${ }^{3}$ (D), Ben Rusholme ${ }^{4}$ (D), David L. Shupe ${ }^{4}$ (D), Maayane T. Soumagnac ${ }^{8}$ (D), and Jeffry Zolkower ${ }^{6}$ \\ ${ }^{1}$ Department of Astronomy, University of Maryland, College Park, MD 20742-0001, USA; msk@astro.umd.edu \\ ${ }^{2}$ Physics Department, Leach Science Center, Auburn University, Auburn, AL 36832, USA \\ ${ }^{3}$ Division of Physics, Mathematics, and Astronomy, California Institute of Technology, Pasadena, CA 91125, USA \\ ${ }^{4}$ Infrared Processing and Analysis Center, California Institute of Technology, Pasadena, CA 91125, USA \\ ${ }^{5}$ DIRAC Institute, Department of Astronomy, University of Washington, 3910 15th Avenue NE, Seattle, WA 98195, USA \\ ${ }^{6}$ Caltech Optical Observatories, California Institute of Technology, Pasadena, CA 91125, USA \\ ${ }^{7}$ Kavli Institute for Theoretical Physics, University of California, Santa Barbara, CA 93106, USA \\ ${ }^{8}$ Benoziyo Center for Astrophysics, Weizmann Institute of Science, Rehovot, Israel \\ Received 2019 July 16; revised 2019 October 22; accepted 2019 November 4; published 2019 November 18
}

\begin{abstract}
Comets are primitive objects that formed in the protoplanetary disk, and have been largely preserved over the history of the solar system. However, they are not pristine, and surfaces of cometary nuclei do evolve. In order to understand the extent of their primitive nature, we must define the mechanisms that affect their surfaces and comae. We examine the lightcurve of comet 240P/NEAT over three consecutive orbits, and investigate three events of significant brightening ( $\Delta m \sim-2 \mathrm{mag}$ ). Unlike typical cometary outbursts, each of the three events are long-lived, with enhanced activity for at least 3-6 months. The third event, observed by the Zwicky Transient Facility, occurred in at least two stages. The anomalous behavior appears to have started after the comet was perturbed by Jupiter in 2007, reducing its perihelion distance from 2.53 to $2.12 \mathrm{au}$. We suggest that the brightening events are temporary transitions to a higher baseline activity level, brought on by the increased insolation, which has warmed previously insulated sub-surface layers. The new activity is isolated to one or two locations on the nucleus, indicating that the surface or immediate sub-surface is heterogeneous. Further study of this phenomenon may provide insight into cometary outbursts, the structure of the near-surface nucleus, and cometary nucleus mantling.
\end{abstract}

Unified Astronomy Thesaurus concepts: Cometary studies (279); Light curves (918)

\section{Introduction}

Cometary nucleus surfaces are dynamic, with many processes affecting their volatile content, strength, particle size distribution, and mass loss (Thomas et al. 2013, 2015; Veverka et al. 2013; El-Maarry et al. 2015; Sunshine et al. 2016). Most processes are ultimately driven by insolation. This fact enables the study of nuclear surfaces through examination of gas and dust production as they rotate and orbit the Sun. The correlation of composition or mass-loss rates with insolation may reveal the composition, structure, or evolution of the near-surface layer (e.g., Biver et al. 1997; Meech et al. 2013; Bodewits et al. 2014; Feaga et al. 2014).

Gradual and repeated variations occur on seasonal and diurnal timescales as localized sources on the nucleus vary in activity (e.g., Hayward et al. 2000; Schleicher 2007; A'Hearn et al. 2011; Kramer et al. 2017). In contrast, cometary outbursts are more stochastic. These impulsive increases in mass-loss rate eject material into the coma, causing an immediate and rapid brightening in telescopic observations. The total brightness of the coma varies with a near-exponential decay as the outburst ejecta slowly leaves the vicinity of the nucleus (Hughes 1990). The causes of outbursts vary (Hughes 1991). For example, they may be driven by sub-surface energy storage (Agarwal et al. 2017), rotationally induced mass shedding (Steckloff \& Samarasinha 2018), cliff collapse (Pajola et al. 2017), and water ice phase state transitions (Prialnik \& BarNun 1990; Belton \& Melosh 2009). On occasion, outbursts signal the complete disruption of the nucleus (Farnham et al. 2001; Knight \& Battams 2014; Li \& Jewitt 2015).
Comet 240P/NEAT is a Jupiter-family comet, discovered in 2002 as P/2002 X2 by the Near-Earth Asteroid Tracking (NEAT) survey with the $1.2 \mathrm{~m}$ Samuel Oschin telescope at Palomar Observatory (Lawrence et al. 2002). With a $7.6 \mathrm{yr}$ orbital period, it has been observed over three perihelion passages. On 2007 July 10, it made a close approach to Jupiter $\left(\Delta_{J}=0.25 \mathrm{au}\right.$, heliocentric distance $\left.r_{\mathrm{h}}=5.5 \mathrm{au}\right)$ and its perihelion distance, $q$, dropped from 2.53 to 2.12 au (NASA JPL Small-Body Database, orbital solution JPL K182/8), corresponding to a $40 \%$ increase in insolation at perihelion. Prior to this encounter, the comet's orbit had been stable with $q$ near 2.5-2.6 au for at least $80 \mathrm{yr}$, according to the same JPL solution.

After passing through perihelion in the newly perturbed orbit, an apparent 2 mag outburst was reported by B. Haeusler, occurring between 2011 March 29 and April 6. On the next perihelion passage, a second apparent 2 mag outburst was reported by Sato (2017), between 2017 July 18.63 and August 28.59 UTC. In 2018, S. Yoshida (2019, personal communication) received a report from $\mathrm{T}$. Ikemura and $\mathrm{H}$. Sato (at Shinshiro, IAU observatory code Q11) that the comet had experienced a third apparent outburst, 1-2 mag in strength, between 2018 November 14.81 and December 12.68 UTC.

We examine the apparent outbursts and baseline activity of comet 240P. We present photometry of the comet from the Zwicky Transient Facility (ZTF; Bellm et al. 2019; Graham et al. 2019), the Palomar Transient Factory (PTF;

\footnotetext{
9 https://groups.yahoo.com/neo/groups/comets-ml/conversations/ messages / 17241
} 
Law et al. 2009; Rau et al. 2009), and the NEAT survey. These data, in combination with photometry reported to the Minor Planet Center (MPC), reveal a comet in repeated transition between two different activity states.

\section{Observations and Results}

The ZTF is a time-domain all-sky survey and successor to the PTF. First light was acquired 2017 November 1, and science operations commenced 2018 March 20. The camera utilizes $166 \mathrm{k} \times 6 \mathrm{k}$ CCDs ( 1 !" 01 per pixel) to cover a 47 square degree field of view. It is mounted on the $1.2 \mathrm{~m}$ Oschin Schmidt telescope at Palomar Observatory. Survey operations typically use $30 \mathrm{~s}$ exposures, allowing ZTF to cover 3800 square degrees an hour to a $5 \sigma$ depth of $r=20-21 \mathrm{mag}$ (Bellm et al. 2019; Graham et al. 2019).

We searched for observations of comet 240P/NEAT in the ZTF Data Release 1 and Partnership data archives (Masci et al. 2019) with the ZChecker program (Kelley et al. 2019). Survey coverage of the comet began on 2018 September 11.51 UTC at $r_{\mathrm{h}}=2.3 \mathrm{au}, 119$ days after perihelion $\left(T_{P}=2018\right.$ May 15.88 UTC). It was also observed on two nights in December 2017 during ZTF's commissioning period. We inspected 63 ZTF $g_{-}, r$-, and $i$-band images covering the comet, and measured its brightness using $15,000 \mathrm{~km}$ radius apertures. Several images were dropped from the analysis for various reasons, including stellar contamination, background artifacts, suspected clouds, or high background. The uniform aperture size was chosen to account for seeing and geocentric distance variations throughout the observation period (minimum aperture is $6 " .9$, median seeing is 2 ". 2 ). Photometry was calibrated to the PanSTARRS (PS1) data release 1 (DR1) catalog (Tonry et al. 2012) using the ZTF pipeline (Masci et al. 2019). We measured $g-r=0.56 \pm 0.02 \mathrm{mag}$ from the average of seven $g$ - and $r$-band image pairs; $r-i$ cannot be directly measured because the $i$-band images are separated from the other images by many days. We assume a constant spectral gradient from $g$ to $i$, i.e., $r-i=0.27 \mathrm{mag}$. We used these values to color correct the data from the ZTF filters to the PS1 system (AB magnitudes). The results are binned by day (Table 1).

We also searched for comet 240P in the PTF archive with an online application at the Infrared Science Archive, and found eight images observed with an $R$-band filter. PTF image processing and photometric calibration is described by Laher et al. (2014) and Ofek et al. (2012). We calibrated the images to PS1 $r$-band magnitudes using background stars and the calviacat program (Kelley \& Lister 2019). Photometry of the comet in 9". 5 radius apertures is presented in Table 1 . The fixed angular size was chosen to make the results more comparable to the MPC photometry (justified in Section 4.1).

In addition, we obtained all comet $240 \mathrm{P}$ photometry reported to the MPC (Williams 2019). The data were taken with a wide range of calibration methods, aperture sizes, and bandpasses. As a result, there is a large scatter in reported magnitudes, even when data are separated into "nuclear" (small) and "total" (whole coma) magnitudes. We select all photometry from a subset of observatories (360, 644, 693, 699, 704, E12, H45, G96, T08, T05, V06, 958, B96, H47, J38, B82, and A71), chosen for broad time coverage and the best-quality data. The remaining MPC data produce an improved lightcurve, but still have scatter at the magnitude level. However, activity trends are apparent in the data, therefore we include them in our analysis.
We also searched the data archived at the Canadian Astronomy Data Centre (Gwyn et al. 2012) for pre-discovery (2002) detections of 240P. The comet was covered by NEAT survey images on 1998 May 3 and May $24\left(r_{\mathrm{h}}=5.4 \mathrm{au}\right)$, but the predicted brightness $(V \sim 21 \mathrm{mag})$ was below the sensitivity limit of the images $(V \sim 19 \mathrm{mag})$. A search by eye on the images within the uncertainty ellipse $\left(<3^{\prime \prime}\right)$ did not turn up any evidence for the object. We conclude that the comet was not more than 2 magnitudes brighter than the 2003 activity level at that time.

Three images of comet 240P on 2003 January 16 were found in the partial NEAT data archive of Bauer \& Lawrence (2013). We bias-subtracted and flat-fielded the data, and measured the coma within $7 "$ radius apertures. The photometric aperture is limited due to a nearby star. The unfiltered images were calibrated to PS1 $r$-band magnitudes using background stars. The weighted-mean photometry is presented in Table 1 .

\section{Models}

To model the comet's photometric behavior, we use the $A f \rho$ coma quantity of A'Hearn et al. (1984). It is proportional to the apparent brightness of the comet, and is defined as the product of grain albedo, filling factor within the aperture, and aperture size projected to the distance of the comet:

$$
A(\theta) f \rho=\frac{4 \Delta^{2} r_{\mathrm{h}}^{2} F_{\lambda}}{\rho S_{\lambda}}
$$

where $A(\theta) f \rho$ specifies that the measurement is for a specific phase angle $\theta, \Delta$ is the observer-comet distance, $F_{\lambda}$ is the observed spectral flux density of the coma within a circular aperture with projected linear radius $\rho$, and $S_{\lambda}$ is the spectral flux density of sunlight at $1 \mathrm{au}$. Despite the units of length, $A f \rho$ is a proxy for the comet's intrinsic dust coma activity, i.e., mass-loss rate (Fink \& Rubin 2012). To model the comet's brightness, we assume $A f \rho$ varies as a power law based on the heliocentric distance $\left(r_{\mathrm{h}}\right)$ :

$$
A(\theta) f \rho=A\left(0^{\circ}\right) f \rho \Phi(\theta)\left(\frac{r_{\mathrm{h}}}{q}\right)^{k},
$$

where $\Phi$ is a phase function for light scattered by cometary dust (Schleicher \& Bair 2011), $q$ is the perihelion distance, and $k$ is the power-law slope. $A\left(0^{\circ}\right) f \rho$ is the value that would be measured if the comet were observed at a phase angle of $0^{\circ}$.

We also interpret the comet's activity state with the ice sublimation model of Cowan \& A'Hearn (1979). This model balances absorption of sunlight by a low-albedo (5\% bond albedo) spherical nucleus with the energy losses of thermal radiation and ice sublimation. Based on spacecraft observations, cometary surfaces have low thermal inertias (Davidsson et al. 2013; Groussin et al. 2013; Gulkis et al. 2015); i.e., their surface temperatures are in near-instantaneous equilibrium with sunlight. We assume the same property for the nucleus of $240 \mathrm{P}$ in our ice sublimation model.

\section{Analysis}

\subsection{Orbit: $T_{P}=2018$ May 15.88 UTC}

The ZTF photometry yields an unusual lightcurve with two apparent brightening events at $T_{P}+172$ and +185 days (Figure 1 ). The first brightening had a strength of $\Delta m \sim-0.7 \mathrm{mag}$, and 
Table 1

Photometry of Comet 240P/NEAT

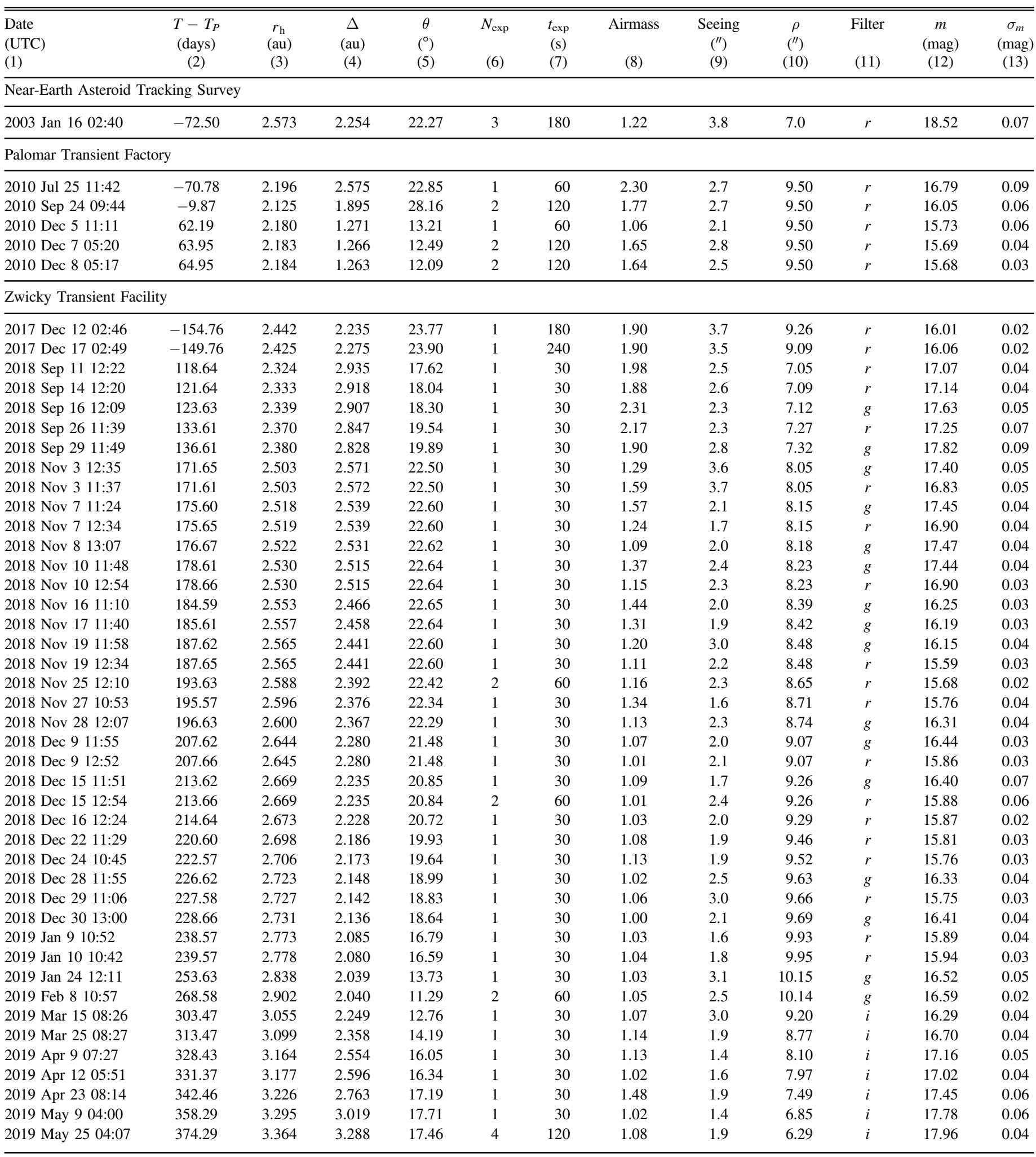

Note. Column definitions: (1) mean observation date; (2) time from nearest perihelion date; (3) heliocentric distance; (4) observer-comet distance; (5) phase angle (Sun-target-observer); (6) number of exposures; (7) total exposure time; (8) mean airmass; (9) mean seeing (stellar FWHM); (10) photometric aperture radius; (11) filter bandpass, data from ZTF $r$ and PTF $R$ and NEAT (unfiltered) are calibrated to PS1 $r$, data from ZTF $g$ are calibrated to PS1 $g$; (12) apparent magnitude; (13) uncertainty on $m$. 


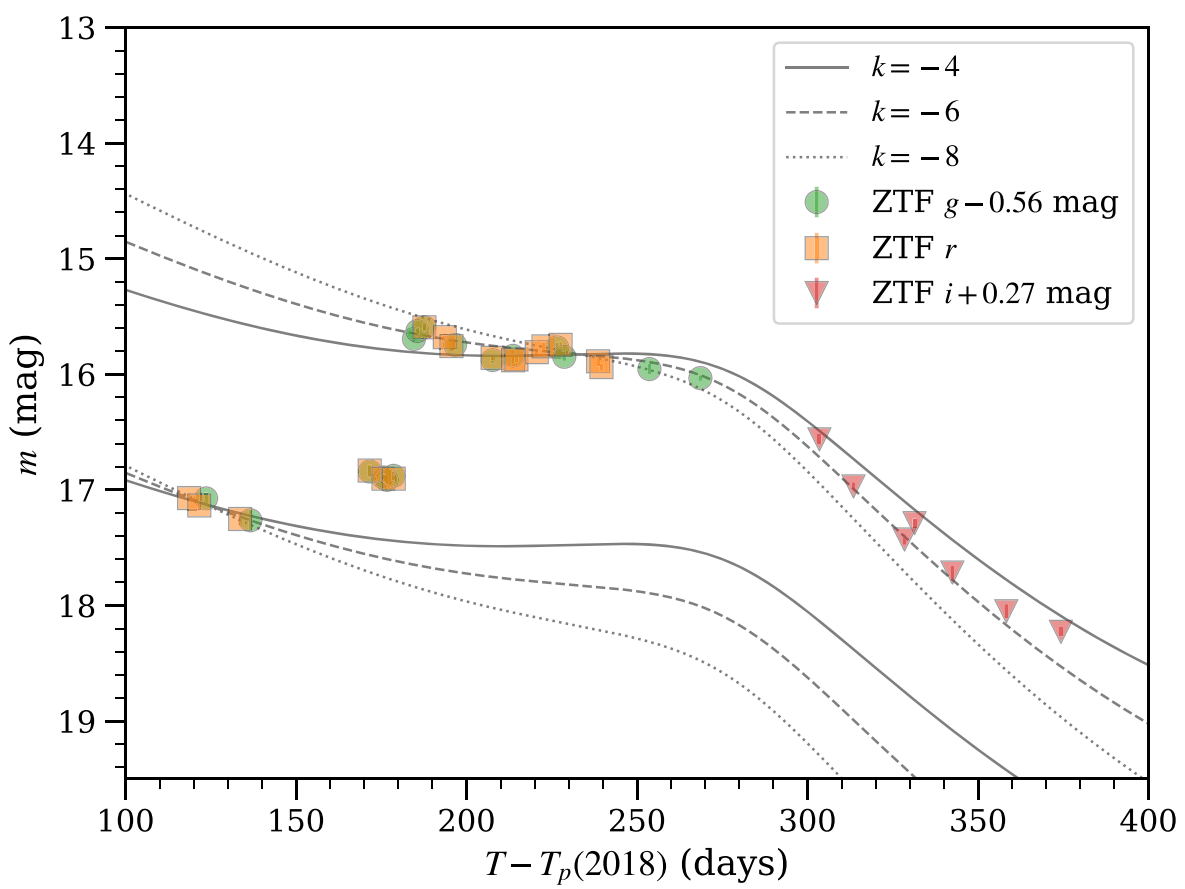

Figure 1. ZTF photometry of comet 240P/NEAT, measured with a 15,000 km radius aperture. Several photometric models are shown to demonstrate our model uncertainty on the heliocentric distance power-law slope, $k$.

occurred between $T_{P}+136.6$ and +171.6 days (September 29-November 3), where $T_{P}$ is the perihelion date. After seven nights of a near-constant apparent magnitude, a second event occurred between $T_{P}+178.7$ and +184.6 days (November 10 and 16), increasing the total brightening to $\Delta m=-2$ mag. The latter event is the 2018 outburst identified by Ikemura and Sato (between $T_{P}+182.9$ and +210.8 days). These data indicate that the full 2 mag event was not simply a gradual increase in activity, but occurred in at least two stages. The apparent magnitude peaks on day three of the second event at +187.6 days. Subsequent structure in the lightcurve suggests a $\Delta m \sim-0.1$ mag peak near $T_{P}+221$ days.

Figure 1 presents lightcurves based on our $A f \rho$ model using the heliocentric distance slopes $k=-4,-6$, and -8 . The lightcurves have the same aperture radius as the ZTF photometry $(15,000 \mathrm{~km})$. The data at $\sim T_{P}+130$ days cannot be used to discriminate between the three slopes (reduced $\chi^{2}$ values are $0.4-0.6)$. However, $k=-6$ is the best of the three, and predicts $A\left(0^{\circ}\right) f \rho=213 \mathrm{~cm}$ at perihelion.

None of our models account for the $\sim 2$ mag increase in activity at $\sim T_{P}+180$ days. Comparing this change in brightness to the $k=-4$ lightcurve implies the comet had a very slow return to quiescence, but even at $T_{P}+350$ days the comet is still -1.3 mag brighter than the model. The $k=-8$ lightcurve suggests the post-event brightening increased with time, up to $2.8 \mathrm{mag}$ by $T_{P}+350$ days. Although there is no a priori requirement that any of these models fit the post-event lightcurve, the middle value, $k=-6$, is most consistent with it, and we adopt this slope for the remainder of the paper.

All three model lightcurves are nearly parallel to the ZTF photometry, which suggests that the event around $T_{P}+180$ days was not a typical outburst, but rather a new sustained activity level. Good agreement with the data is obtained with the $k=-6$ model for $A\left(0^{\circ}\right) f \rho(q)=1346 \mathrm{~cm}$ (Figure 1), equivalent to a factor of 6 increase in the dust production rate.
We examined the ZTF images to determine if the sustained brightening was due to lingering large grains, a new fragment, or to new activity. Images were averaged into three bins to enhance the data quality (pre-event: 2018 September 11-29 (five images); early-event: November 16-19 (five images); mid-event: 2019 February 2 (one image); late-event: March 15-April 9 (three images)). The results and azimuthally averaged profiles are presented in Figure 2. There is no morphological evidence for a new fragment. Before the event, the radial profile was close to $\rho^{-1.5}$, the canonical distribution of a tail-dominated image (Jewitt \& Meech 1987). Immediately after the event, the profile was steeper than $\rho^{-1.5}$, which is indicative of an outburst early in its evolution when the ejecta is close to the nucleus. The radial profile returns to the preevent distribution in the mid- and late-event images. An impulsive event cannot simultaneously have a $\rho^{-1.5}$ profile and a consistently high intrinsic brightness over this 130 day period, unless it were accompanied with new activity.

Figure 3 presents the long-term lightcurve of the comet based on the ZTF, PTF, and MPC photometry. Here, the ZTF photometry has been remeasured with a 9!"5 radius aperture. This choice of aperture size produced photometry in agreement with the data reported by the Asteroid Terrestrial-impact Last Alert System (ATLAS) survey (Tonry et al. 2018), which accounts for most of the 2017/2018 photometry and informed our PTF photometric aperture choice. For this aperture size, the 2018 events increased the coma $A f \rho$ by a factor of $\sim 9$. The discrepancy with Figure 1, where a factor of $\sim 6$ change was found, is due to the limitations of the $A f \rho$ model, which assumes a $1 / \rho$ surface brightness distribution.

Inspection of the pre-perihelion MPC data reveals the apparent outburst identified by Sato (2017) began between two sets of observations by ATLAS, at $T_{P}-272.3$ and -270.3 days (2018 August 16 and 18). The ATLAS photometry suggest an outburst strength of 2-3 mag, followed by a 1-1.5 mag decay in 14 days. However, no ATLAS photometry 


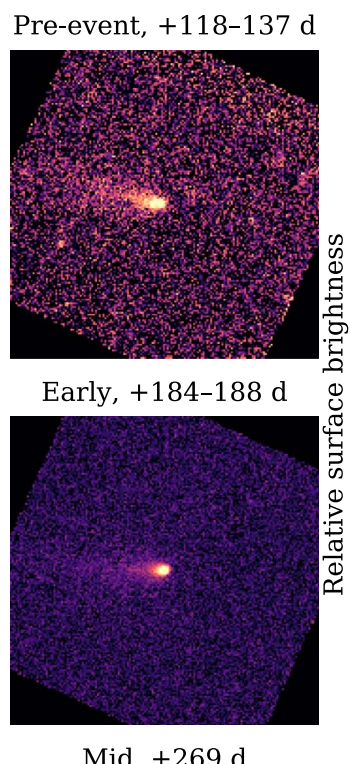

Mid, +269 d

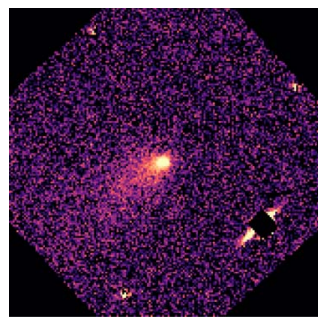

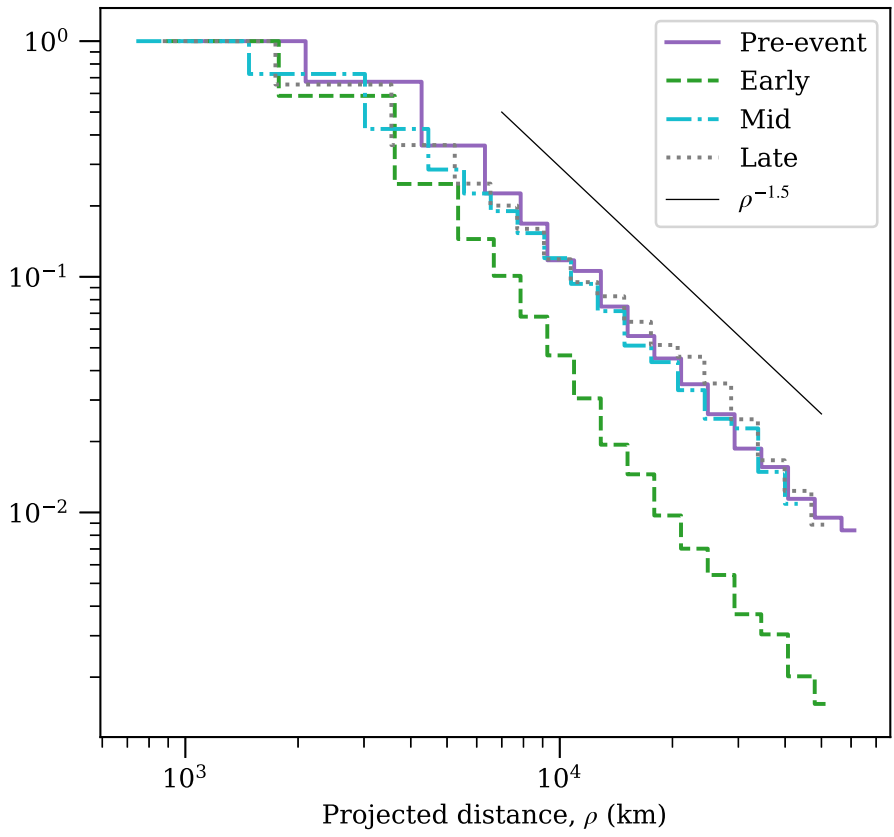

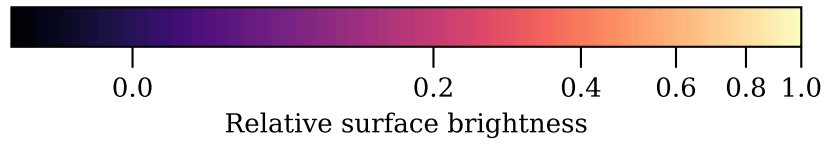

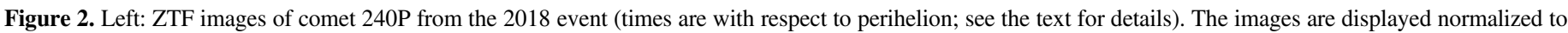

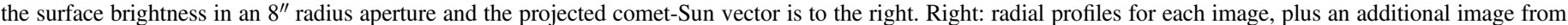
the late-event data $\left(T-T_{p}=303-328\right.$ days). Note that the first three bins are $1^{\prime \prime}, 2^{\prime \prime}$, and $3^{\prime \prime}$.

exists during this decay period. After the initially rapid fading, the coma takes around 80 days to reach the pre-outburst activity level, and continues to fade through the last data at 150 days after the outburst. This timescale is an order of magnitude longer than a typical cometary outburst (Hughes 1990). If the first ATLAS photometry points can be confirmed, it appears the event started with a typical outburst, but ended with an usually long fading period.

The pre- and post-perihelion events are separated by $220^{\circ}$ of true anomaly. Based on solar illumination of the nucleus, it is possible that a single active area is responsible for both.

\subsection{Orbit: $T_{P}=2010$ October 04.27 UTC}

An activity model with $A\left(0^{\circ}\right) f \rho=150 \mathrm{~cm}$ at perihelion has good agreement with our PTF photometry, and parallels most of the MPC data in 2010-2011 (Figure 3). The outburst reported by Haeusler is clear in the MPC lightcurve at $T_{P}+182$ days. The data suggest the comet took 20 days to reach peak brightness, after which the coma remains near the $1350 \mathrm{~cm}$ model for 90 days, up to the last reported data for this period. This is the same part of the orbit as the 2018 brightening; both have on-set dates near +180 days. The portion of the orbit that covers the pre-perihelion event $\left(T_{P}-270\right.$ to -120 days $)$ was not observed.

\subsection{Orbit: $T_{P}=2003$ March 29.61 UTC}

The MPC photometric coverage in 2002-2004 covers $T_{P}-180$ to -60 days, and +247 to +353 days. We examined these data for evidence of the 2011, 2017, and 2018 events.
Because the orbit changed after 2003, we plot the lightcurve versus ecliptic longitude of the comet-Sun vector, $\lambda_{\odot}$, under the assumption that the events are tied to specific illumination conditions on the nucleus. Figure 4 compares the ZTF photometry to the 2003 MPC and NEAT data using absolute magnitude:

$$
H(1,1,0)=m-5 \log _{10}\left(r_{\mathrm{h}} \Delta\right)-2.5 \log _{10}(\Phi(\theta)),
$$

where $\Phi$ is the Halley-Marcus phase function from Schleicher $\&$ Bair (2011) evaluated at phase angle $\theta$. Furthermore, the ZTF data are scaled to the 2003 circumstances using Equation (1). The pre-perihelion event would have spanned $\lambda_{\odot}=165^{\circ}-205^{\circ}$, and its presence in 2003 cannot be tested. The post-perihelion events observed in 2011 and 2018 would have spanned from $\lambda_{\odot}=305^{\circ}$ to at least $350^{\circ}$. Had this brightening occurred in the 2003 orbit, then we should have seen a comet near 18-19 mag, rather than the observed 20-21 mag. Either the event did not occur at that time, or it was much smaller in strength.

The 2003 lightcurve roughly agrees with $A\left(0^{\circ}\right) f \rho \sim 40 \mathrm{~cm}$ for $k=-6$ (Figure 4). A model following $A\left(0^{\circ}\right) f \rho \sim 38 \mathrm{~cm}$ for $k=-4$ (not shown) fits equally well. The lower activity level of this orbit is caused by the change in perihelion distance between 2003 and 2010. Scaling the 2010 estimate, $A\left(0^{\circ}\right) f \rho \sim 150 \mathrm{~cm}$, by $(2.53 / 2.12)^{-6}$ yields $52 \mathrm{~cm}$ at perihelion.. This factor of 3 change is larger than the factor of 2 predicted by the water ice sublimation model of Cowan \& A'Hearn (1979). 

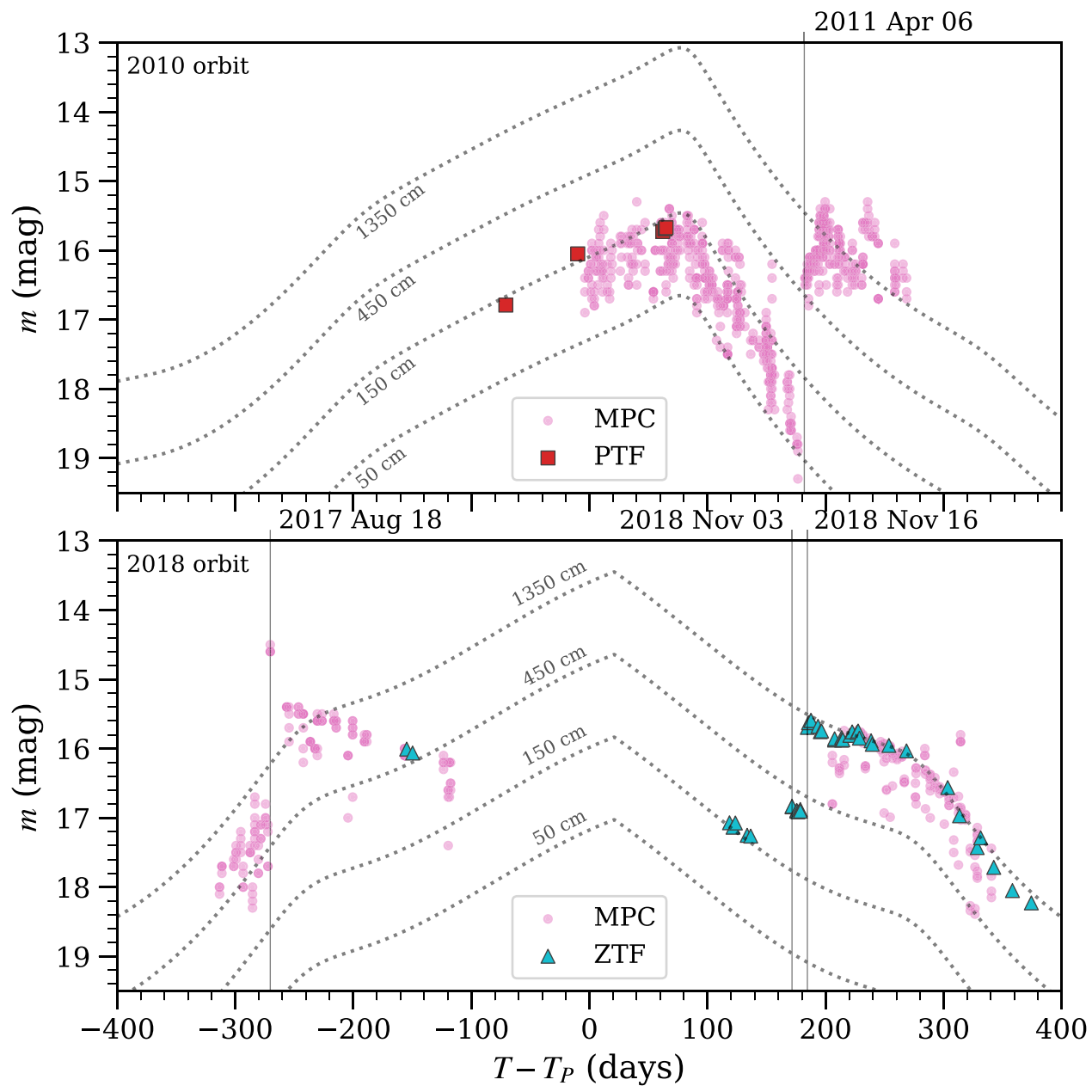

Figure 3. Lightcurve of comet 240P/NEAT vs. perihelion time $\left(T-T_{P}\right)$ in 2010-2011 (top) and 2017-2018 (bottom). ZTF and PTF data are measured with 9". 5 radius apertures. Select photometry contributed to the MPC are also shown (see Section 2 for details). Model lightcurves are presented (dotted lines), based on the $A f \rho$ formalism using the indicated value at perihelion $\left(9{ }^{\prime \prime} 5\right.$ aperture, $r_{\mathrm{h}}^{-6}$ scale factor). Approximate epochs of transition to increased activity levels are marked with vertical lines.

\section{Discussion}

The comet's behavior appears to have changed after the 2007 gravitational perturbation by Jupiter. In 2010, the comet returned to perihelion with a peak activity level nearly consistent with the reduced perihelion distance. 180 days after perihelion, the comet brightened by $\sim-2 \mathrm{mag}$, and remained bright for at least 90 days. In 2017, the comet again brightened by $\sim-2$ mag, but this time slowly returned to a quiescent state, with a timescale much longer than is typical for outbursts, 150 versus 20-30 days (Hughes 1990). A third -2 mag brightening was observed in 2018, occurring in at least two stages over a 50 day period. The comet remained at this new activity level for 190 days, up to the end of our data set. Given these observations, we identify the following features of interest.

1. The comet brightened three times over two orbits, achieving nearly the same peak activity level each time: $A\left(0^{\circ}\right) f \rho \sim 1350 \mathrm{~cm}$, corrected for heliocentric distance. This behavior is unusual for cometary outbursts at shortperiod comets, which have a power-law distribution in total mass (Ishiguro et al. 2016). To have three large events of the same order of magnitude is an indication that the same active area may be responsible for all events. A better understanding of this repeatability may provide insight into possible outburst trigger mechanisms, or the near-surface structure of the active area in question.

2. The three brightening events all occurred after the 2007 orbital perturbation by Jupiter, which increased surface insolation at perihelion by $40 \%$ and the dust production rate by at least a factor of 3 . The data from the 2003 orbit are sparse, but there is no evidence for anomalous behavior. Perhaps moderate changes in orbits can have profound consequences on cometary mass loss.

3. Two of the brightening events occurred near the same point in the orbit on two separate orbits, near $T_{P}+180$ days in 2011 and 2018. Both events are long lived, and the comet remains at the higher activity level for at least several months. However, there is a lack of a similar event in 2003. A single active area may be responsible for all events, and appears to have been in relative quiescence in 2003.

We propose a scenario to account for these observations. The pre-2007 comet was near a steady state, balancing sublimation driven erosion with sub-surface devolatilization. The perihelion distance change perturbed this scenario, and warmed volatilerich sub-surface layers that were previously insulated from the 


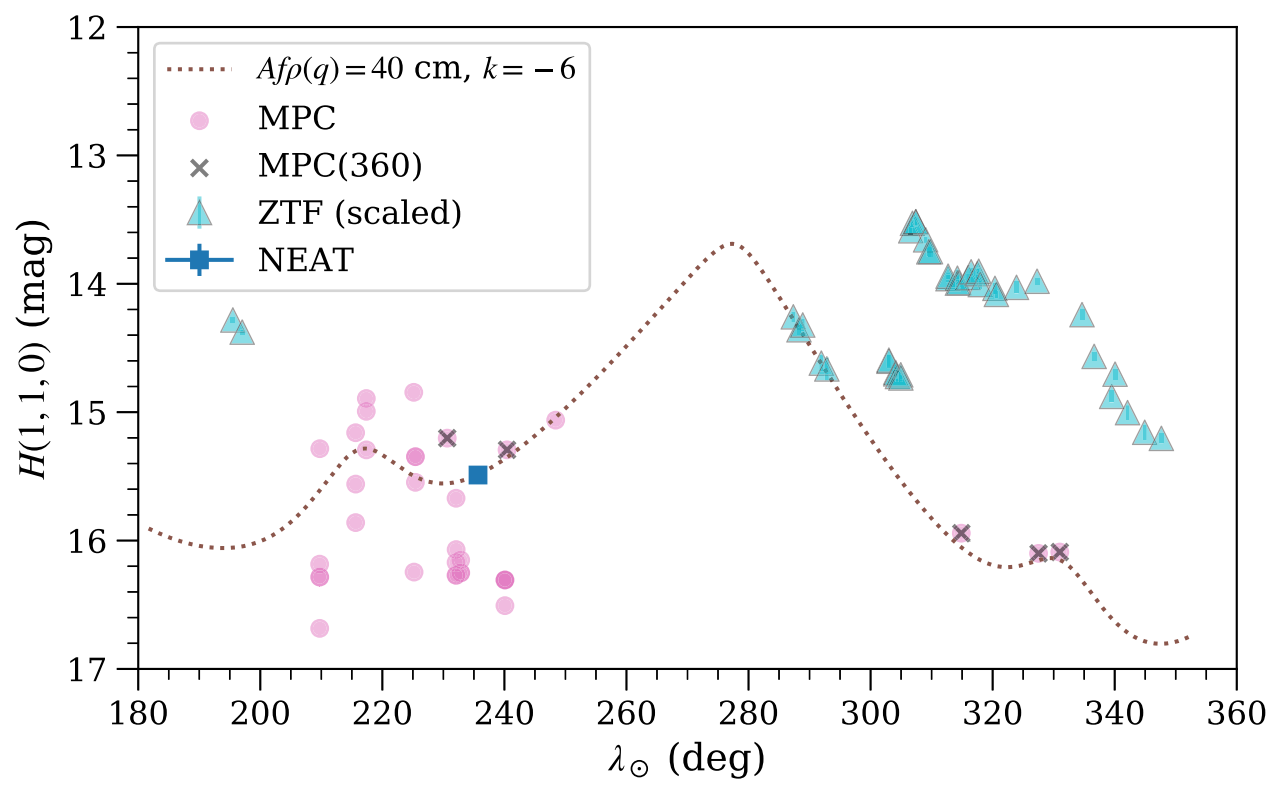

Figure 4. Absolute magnitude of comet 240P/NEAT from 2003 vs. ecliptic longitude of the comet-Sun vector. NEAT photometry within a 7 " radius aperture, and select MPC photometry is shown. Photometry from Kuma Kogen Astronomical Observatory (MPC code 360), which observed both sides of perihelion, is highlighted. ZTF photometry from 2017 to 2018 is also shown, scaled to match the observation circumstances of 2003 . The model lightcurve ( 7 " aperture, $r_{\mathrm{h}}^{-6}$ scale factor) was fit to the NEAT data point.

thermal wave. The new activity is isolated to one or two locations on the nucleus, indicating that the surface or immediate sub-surface is heterogeneous.

Terrain and activity heterogeneities are commonly observed by spacecraft missions to comets (A'Hearn et al. 2005, 2011; Veverka et al. 2013; Hässig et al. 2015; Thomas et al. 2015). The first observed transition (2010 orbit, $T_{P}+182$ days) appears to have rejuvenated a local active area, perhaps shedding off an insulating layer. We speculate that the same surface is illuminated upon the approach to perihelion. Thus, the surface renewal may have occurred during the unobserved pre-perihelion approach in 2009. Tests of this idea would benefit from a pole orientation measurement. Thermophysical modeling of these events, and photometric observations during future perihelion passages will also help explore our proposed scenario.

Short-period comets, such as comet 240P, provide examples of cometary evolution from the cumulative effects of perihelion passages and orbital perturbations. After perturbations to smaller perihelion distances, comets may have a greater tendency toward enhanced activity levels (cometary rejuvenation), or the volatile reservoirs may quickly diminish (rapid surface mantling or devolatilization). Archival searches for comets under similar circumstances, and future comparisons to data taken with present-day surveys would benefit the study of comet behavior in general. We observed comet $240 \mathrm{P}$ at an interesting moment in its evolution. The pre-perihelion portion of the comet's 2025 orbit should be well observed with the Large Synoptic Survey Telescope, with science operations expected to begin in 2023 .

We thank all amateur astronomers contributing to the discovery of cometary outbursts.

Based on observations obtained with the Samuel Oschin Telescope 48 inch at the Palomar Observatory as part of the Zwicky Transient Facility project. Z.T.F. is supported by the National Science Foundation under grant No. AST-1440341 and a collaboration including Caltech, IPAC, the Weizmann Institute for Science, the Oskar Klein Center at Stockholm University, the University of Maryland, the University of Washington, Deutsches Elektronen-Synchrotron and Humboldt University, Los Alamos National Laboratories, the TANGO Consortium of Taiwan, the University of Wisconsin at Milwaukee, and Lawrence Berkeley National Laboratories. Operations are conducted by COO, IPAC, and UW.

Facilities: PO:1.2 m (NEAT, PTF, ZTF).

Software: Astropy (Astropy Collaboration et al. 2018), SEP (Barbary 2016), ZChecker (Kelley et al. 2019), Calviacat (Kelley \& Lister 2019).

\section{ORCID iDs}

Michael S. P. Kelley (1) https://orcid.org/0000-00026702-7676

Dennis Bodewits (1) https://orcid.org/0000-0002-2668-7248

Quanzhi Ye (叶泉志) (1) https://orcid.org/0000-0002-4838-7676

Tony L. Farnham (i) https://orcid.org/0000-0002-4767-9861

Eric C. Bellm @ https://orcid.org/0000-0001-8018-5348

Dmitry A. Duev (i) https://orcid.org/0000-0001-5060-8733

George Helou (1) https://orcid.org/0000-0003-3367-3415

Thomas Kupfer (ii) https://orcid.org/0000-0002-6540-1484

Russ R. Laher (1) https://orcid.org/0000-0003-2451-5482

Frank J. Masci $\odot$ https://orcid.org/0000-0002-8532-9395

Thomas A. Prince (1) https://orcid.org/0000-0002-8850-3627

Ben Rusholme 주 https://orcid.org/0000-0001-7648-4142

David L. Shupe 다 https://orcid.org/0000-0003-4401-0430

Maayane T. Soumagnac (i) https://orcid.org/0000-00016753-1488

\section{References}

Agarwal, J., Della Corte, V., Feldman, P. D., et al. 2017, MNRAS, 469, s606 A'Hearn, M. F., Belton, M. J. S., Delamere, W. A., et al. 2005, Sci, 310, 258 A'Hearn, M. F., Belton, M. J. S., Delamere, W. A., et al. 2011, Sci, 332,1396 
A'Hearn, M. F., Schleicher, D. G., Millis, R. L., Feldman, P. D., \& Thompson, D. T. 1984, AJ, 89, 579

Astropy Collaboration, Price-Whelan, A. M., Sipőcz, B. M., et al. 2018, AJ, 156,123

Barbary, K. 2016, JOSS, 1, 58

Bauer, J. M., \& Lawrence, K. J. 2013, Near Earth Asteroid Tracking V1.0, EAR-A-I1063-3-NEAT-V1.0, NASA Planetary Data System

Bellm, E. C., Kulkarni, S. R., Graham, M. J., et al. 2019, PASP, 131, 018002

Belton, M. J. S., \& Melosh, J. 2009, Icar, 200, 280

Biver, N., Bockelee-Morvan, D., Colom, P., et al. 1997, Sci, 275, 1915

Bodewits, D., Farnham, T. L., A'Hearn, M. F., et al. 2014, ApJ, 786, 48

Cowan, J. J., \& A'Hearn, M. F. 1979, M\&P, 21, 155

Davidsson, B. J. R., Gutiérrez, P. J., Groussin, O., et al. 2013, Icar, 224, 154

El-Maarry, M. R., Thomas, N., Giacomini, L., et al. 2015, A\&A, 583, A26

Farnham, T. L., Schleicher, D. G., Woodney, L. M., et al. 2001, Sci, 292, 1348

Feaga, L. M., A'Hearn, M. F., Farnham, T. L., et al. 2014, AJ, 147, 24

Fink, U., \& Rubin, M. 2012, Icar, 221, 721

Graham, M. J., Kulkarni, S. R., Bellm, E. C., et al. 2019, PASP, 131, 078001

Groussin, O., sunshine, J. M., Feaga, L. M., et al. 2013, Icar, 222, 580

Gulkis, S., Allen, M., von Allmen, P., et al. 2015, Sci, 347, aaa0709

Gwyn, S. D. J., Hill, N., \& Kavelaars, J. J. 2012, PASP, 124, 579

Hässig, M., Altwegg, K., Balsiger, H., et al. 2015, Sci, 347, aaa0276

Hayward, T. L., Hanner, M. S., \& Sekanina, Z. 2000, ApJ, 538, 428

Hughes, D. W. 1990, QJRAS, 31, 69

Hughes, D. W. 1991, in IAU Coll. 116: Comets in the post-Halley Era 167, Astrophysics and Space Science Library, ed. R. L. Newburn, Jr., M. Neugebauer, \& J. Rahe (Dordrecht: Kluwer), 825

Ishiguro, M., Kuroda, D., Hanayama, H., et al. 2016, AJ, 152, 169

Jewitt, D. C., \& Meech, K. J. 1987, ApJ, 317, 992
Kelley, M. S. P., Bodewits, D., Ye, Q., et al. 2019, in ASP Conf. Ser. 523, Astronomical Data Analysis Software and Systems XXVIII, ed. P. Teuben (San Francisco: ASP)

Kelley, M. S. P., \& Lister, T. 2019, Calviacat: Calibrate Star Photometry by Comparison to a Catalog, v1.0.2, Zenodo, doi:10.5281/zenodo.2635841

Knight, M. M., \& Battams, K. 2014, ApJL, 782, L37

Kramer, T., Läuter, M., Rubin, M., \& Altwegg, K. 2017, MNRAS, 469, S20 Laher, R. R., Surace, J., Grillmair, C. J., et al. 2014, PASP, 126, 674

Law, N. M., Kulkarni, S. R., Dekany, R. G., et al. 2009, PASP, 121, 1395 Lawrence, K. J., Bambery, R., Helin, E., et al. 2002, IAU Circ., 8029, 1 Li, J., \& Jewitt, D. 2015, AJ, 149, 133

Masci, F. J., Laher, R. R., Rusholme, B., et al. 2019, PASP, 131, 018003

Meech, K. J., Yang, B., Kleyna, J., et al. 2013, ApJL, 776, L20

Ofek, E. O., Laher, R., Law, N., et al. 2012, PASP, 124, 62

Pajola, M., Höfner, S., Vincent, J. B., et al. 2017, NatAs, 1, 0092

Prialnik, D., \& Bar-Nun, A. 1990, ApJ, 363, 274

Rau, A., Kulkarni, S. R., Law, N. M., et al. 2009, PASP, 121, 1334

Sato, H. 2017, CBET, 4427, 1

Schleicher, D. G. 2007, Icar, 190, 406

Schleicher, D. G., \& Bair, A. N. 2011, AJ, 141, 177

Steckloff, J. K., \& Samarasinha, N. H. 2018, Icar, 312, 172

Sunshine, J. M., Thomas, N., El-Maarry, M. R., \& Farnham, T. L. 2016, JGRE, 121,2194

Thomas, N., Sierks, H., Barbieri, C., et al. 2015, Sci, 347, aaa0440

Thomas, P., A'Hearn, M., Belton, M. J. S., et al. 2013, Icar, 222, 453

Tonry, J. L., Denneau, L., Heinze, A. N., et al. 2018, PASP, 130, 064505

Tonry, J. L., Stubbs, C. W., Lykke, K. R., et al. 2012, ApJ, 750, 99

Veverka, J., Klaasen, K., A'Hearn, M., et al. 2013, Icar, 222, 424

Williams, G. V. 2019, MPEC, 2019-J142, 1 